\title{
Efficient Locality-Sensitive Hashing Over High-Dimensional Streaming Data
}

\author{
Hao Wang • Chengcheng Yang • \\ Xiangliang Zhang · Xin Gao
}

Received: / Accepted:

\begin{abstract}
Approximate nearest neighbor (ANN) search in high-dimensional spaces is fundamental in many applications. Locality-sensitive hashing (LSH) is a well-known methodology to solve the ANN problem. Existing LSH-based ANN solutions typically employ quite a number of individual indexes optimized for searching efficiency. Updating such indexes might be impractical when processing high-dimensional streaming data. In this paper, we present a novel disk-based LSH index that offers efficient support for both searches and updates. The contributions of our work are threefold. First, we use the write-friendly LSM-trees to store the LSH projections to facilitate efficient updates. Second, we develop a novel estimation scheme to estimate the number of required LSH functions, with which the disk storage and access costs are effectively reduced. Third, we exploit both the collision number and the projection distance to improve the efficiency of candidates selection, improving the search performance with theoretical guarantees on the result quality. Experiments on four real-world datasets show that our proposal outperforms the state-of-the-art schemes on both search and update performance.
\end{abstract}

\footnotetext{
Hao Wang

King Abdullah University of Science and Technology

E-mail: wanghao.hku@gmail.com

Chengcheng Yang

King Abdullah University of Science and Technology

E-mail: chengcheng.yang@kaust.edu.sa

Xiangliang Zhang

King Abdullah University of Science and Technology

E-mail: xiangliang.zhang@kaust.edu.sa

Xin Gao

King Abdullah University of Science and Technology

E-mail: xin.gao@kaust.edu.sa
} 
Keywords Approximate nearest neighbor search • Locality-sensitive hasing • LSM-tree $\cdot$ Streaming data

\section{Introduction}

Nearest neighbor (NN) search in high-dimensional Euclidean space plays an essential role in applications from many domains, including multimedia databases, recommender systems, statistical machine learning, etc. In such applications, data objects are typically characterized as feature vectors/points in multi-dimensional spaces. There are numerous studies on NN search in multidimensional spaces, yet it has been shown that the performance sharply degenerates as the dimensionality increases [1]. Thus, approximate nearest neighbor (ANN) search has been receiving much attention, as it can be much more efficient with theoretically guaranteed quality of search results.

Locality-sensitive hashing ( $\mathrm{LSH})$ [2] is a widely used technique that has shown significant promise in high-dimensional ANN search. LSH solves the c-approximate nearest neighbor (c-ANN) problem, which, given a query object $\boldsymbol{q}$, aims at finding an object $\boldsymbol{o}$ in the database such that the distance from $\boldsymbol{o}$ to $\boldsymbol{q}$ is at most $c$ times the distance from $\boldsymbol{q}$ to its nearest neighbor. As LSH was originally designed to find objects within a fixed distance radius, the basic version of LSH for $c$-ANN has to build thousands of hash tables for different radii, which could be expensive and inefficient. Hence, many LSH variants have been proposed, aiming at building indexes with smaller storage and search costs while preserving acceptable accuracy [3-10]. Using different ways of generating the hash functions, existing studies have developed datadependent and data-independent methods.

Given sufficient training data, data-dependent methods focus on learning a set of hash functions from the density patterns of the data [3-6]. In this work, we argue that such density patterns in streaming data might change over time $[11,12]$. Timely updates to the learned hash functions are crucial but non-trivial, as it often involves expensive learning processes over all the so-far seen data to tune the hash functions. In contrast, data-independent methods use random projections to construct distance-preserving hash functions. In principle, two data points are more likely to be neighboring if they are hashed into the same bucket by more independent LSH functions. Hence, data-independent methods deliberately deal with the tradeoff between the maintenance cost of independent projections and the search accuracy [7]. Sun et al. [10] propose to project high-dimensional data into a low-dimensional space so that multi-dimensional indexes, such as R-tree, can be used. Gan et al. [8] and Huang et al. [9] both store the projections of each LSH function in a separate B-tree. By performing B-tree range searches, their techniques gradually enlarge the query radius to find the $c$-ANN result. However, we observe that updating R-trees or B-trees for streaming data (i.e., adding the projections of the incoming data into the trees) involves random writes to the disk, which could be inefficient. In summary, existing data-independent methods 
cannot make a good trade-off between update efficiency, search efficiency and quality guarantees, but all of which are crucial factors for efficiently indexing high-dimensional data streams.

In this paper, we propose a novel disk-based LSH index that offers efficient $c$-ANN searches with low update cost and guaranteed result quality. We employ a set of individual LSH functions and organize the projections generated by each function in a log-structured merge-tree (LSM-tree). The advantage is that LSM-trees support efficient cascading updates. We estimate the binomial distribution of collision/non-collision using a normal distribution, resulting in a reduced number of required LSH functions. Then, we consider both the collision number and the projection distance in the search process. Specifically, with properly chosen thresholds $L$ and $\Delta, \boldsymbol{o}$ is a good $c$-ANN candidate of $\boldsymbol{q}$ if (1) a data object $\boldsymbol{o}$ collides with the query $\boldsymbol{q}$ under at least $L$ LSH functions and (2) the sum of the $L$ projected distances does not exceed $\Delta$. Comparing to existing solutions, the additional consideration on projection distances typically improves the search efficiency. We shall refer to our proposal as PDA-LSH

(Projection-Distance-Aware LSH).

In summary, our main contributions are the following.

- We propose PDA-LSH, a novel and practical LSH index, to offer efficient support for both searches and updates.

- We use normal distribution to estimate the number of required LSH functions. Compared to the alternatives in existing solutions, our estimation results in much fewer LSH functions and thus higher space efficiency.

- With PDA-LSH, we develop an efficient and effective $c$-ANN search method with guaranteed success probability.

- Extensive experiments on four real-world datasets show that, comparing to existing methods, our proposal is up to 2 times more efficient on searching and 10 times more efficient on updating.

The rest of the paper is organized as follows. Section 2 introduces the preliminaries and related work. Section 3 presents the indexing and search methods of PDA-LSH. Then, Section 4 explains how to set the internal parameters of PDA-LSH. Section 5 experimentally evaluates PDA-LSH. Finally, Section 6 concludes the paper.

\section{Preliminaries and Related Work}

\subsection{Problem Setting}

Consider a database $\mathcal{D}$ with $d$-dimensional data and a query object $\boldsymbol{q}$. Suppose $\boldsymbol{o}^{*} \in \mathcal{D}$ is the exact nearest neighbor of $\boldsymbol{q}$. The target of the $c$-ANN search is to return an object $\boldsymbol{o} \in \mathcal{D}$ satisfying $\operatorname{dist}(\boldsymbol{o}, \boldsymbol{q}) \leq c \cdot \operatorname{dist}\left(\boldsymbol{o}^{*}, \boldsymbol{q}\right)$, where $\operatorname{dist}(\cdot, \cdot)$ denotes the distance between two objects and $c>1$ is the approximation ratio. It can be further extended to $c-k$-ANN search, of which the target is to output $k$ data objects $\boldsymbol{o}_{1}, \boldsymbol{o}_{2}, \cdots, \boldsymbol{o}_{k} \operatorname{such}$ that $\operatorname{dist}\left(\boldsymbol{o}_{i}, \boldsymbol{q}\right) \leq c \cdot \operatorname{dist}\left(\boldsymbol{o}_{i}^{*}, \boldsymbol{q}\right)$ for $i=1,2, \cdots, k$, where $\boldsymbol{o}_{i}^{*}$ is the actual $i$-th nearest neighbor of $\boldsymbol{q}$. 
In this paper, we consider the Euclidean distance and focus on the external memory setting, where both the data and the index reside in external memory. We tackle the problem of solving the $c$-ANN search over high-dimensional data streams, in which case the update efficiency, search efficiency, and quality guarantees are all crucial. We aim to propose an index that not only has low maintenance overhead, but also can provide efficient $c$-ANN searches with guaranteed success probability.

\subsection{Locality-Sensitive Hashing}

LSH was originally designed to solve the $(R, c)$-NN problem, which, given a query $\boldsymbol{q}$, aims to do the following:

- If there exists $\boldsymbol{o} \in \mathcal{D}$ such that $\operatorname{dist}(\boldsymbol{o}, \boldsymbol{q}) \leq R$, then return an object $\boldsymbol{o}^{\prime} \in \mathcal{D}$ satisfying $\operatorname{dist}\left(\boldsymbol{o}^{\prime}, \boldsymbol{q}\right) \leq c R$.

- If $\operatorname{dist}(\boldsymbol{o}, \boldsymbol{q})>c R$ for all $\boldsymbol{o} \in \mathcal{D}$, then return nothing.

The basic idea of LSH is to hash objects within a given distance into the same bucket with high probability, thus preserving the locality information of data in the original space. To support efficient $(R, c)-\mathrm{NN}$ search, LSH usually uses a set of independent random hash functions $\mathcal{H}$ (called the LSH family) to build multiple hash tables. Only objects that collide with the query under sufficiently many hash functions are considered as candidates.

For a domain $\mathbb{R}^{d}$ of the object set, a hash function in an LSH family is a mapping from $\mathbb{R}^{d}$ to some universe $U$. Formally, the sensitivity of an LSH family to locality can be measured as follows:

Definition 1 For any constant $c>1$ and probabilities $p_{1}>p_{2}$, an LSH family $\mathcal{H}=\left\{h: \mathbb{R}^{d} \rightarrow U\right\}$ is called $\left(r, c r, p_{1}, p_{2}\right)$-sensitive if any $\boldsymbol{u}, \boldsymbol{v} \in \mathbb{R}^{d}$ satisfy

$-\operatorname{Pr}_{\mathcal{H}}[h(\boldsymbol{u})=h(\boldsymbol{v})] \geq p_{1}$ when $\operatorname{dist}(\boldsymbol{u}, \boldsymbol{v}) \leq r ;$ and

$-\operatorname{Pr}_{\mathcal{H}}[h(\boldsymbol{u})=h(\boldsymbol{v})] \leq p_{2}$ when $\operatorname{dist}(\boldsymbol{u}, \boldsymbol{v})>c r$.

There have been numerous studies on LSH families for different distance metrics [13-15]. In particular, Datar et al. [15] proposed an LSH family $\mathcal{H}$ for $l_{p}$ metrics based on $p$-stable distributions. Formally, the proposed hash function is defined as follows:

$$
h_{\boldsymbol{a}, b}(\boldsymbol{o})=\left\lfloor\frac{\boldsymbol{a}^{\top} \boldsymbol{o}+b}{w}\right\rfloor .
$$

Here, $\boldsymbol{o} \in \mathbb{R}^{d}$ is a data object; $\boldsymbol{a}$ is a $d$-dimensional vector with each entry chosen independently from a $p$-stable distribution; $w$ is the pre-specified bucket width; and $b$ is a random variable drawn uniformly from the range $[0, w)$. Specially, for the Euclidean distance (i.e., $p=2$ ), the standard normal distribution is a $p$-stable distribution. Given two objects $\boldsymbol{u}$ and $\boldsymbol{v}$ with Euclidean distance 
$\operatorname{dist}(\boldsymbol{u}, \boldsymbol{v})=s$, the probability that $\boldsymbol{u}$ and $\boldsymbol{v}$ collide under a hash function $h_{\boldsymbol{a}, b}$ randomly selected from $\mathcal{H}$ can be computed as [15]:

$$
p(s)=\operatorname{Pr}\left[h_{\boldsymbol{a}, b}(\boldsymbol{u})=h_{\boldsymbol{a}, b}(\boldsymbol{v})\right]=\int_{0}^{w} \frac{2}{s} \varphi\left(\frac{t}{s}\right)\left(1-\frac{t}{s}\right) d t,
$$

where $\varphi(\cdot)$ is the PDF of the standard normal distribution $\mathcal{N}(0,1)$. When $w$ is fixed, $p(s)$ decreases monotonically with $s$. Therefore, by definition, the family of hash functions $h_{\boldsymbol{a}, b}$ is $(r, c r, p(r), p(c r))$-sensitive.

To support efficient $(R, c)$-NN search, LSH usually applies a concatenation of multiple hash functions to build the hash table, where each hash function is randomly selected from the $(R, c R, p(R), p(c R))$-sensitive family. Only objects that collide with the query object $\boldsymbol{q}$ under all the hash functions are considered as candidates, which effectively reduces the number of irrelevant objects (i.e., false positives). Moreover, to raise the success probability of finding the true result that is within distance $R$ from $\boldsymbol{q}$, multiple independent hash tables are built and the union results are returned. The numbers of concatenated hash functions and parameters and independent hash tables should be carefully set to ensure that the $(R, c)$-NN problem can be solved with guaranteed success probability.

\subsection{Related Work}

E2LSH [15] is the most common LSH implementation that supports ANN searches in the Euclidean space. It builds physical hash tables for a series of search radii to solve $(R, c)$-NN problems with varying $R$, which could be space-consuming. To address this issue, virtual rehashing is used in recent developments such as LSB-tree/LSB-forest [16], C2LSH [8], and QALSH [9]. LSB-tree/LSB-forest [16] projects objects into the space $\mathcal{Z}^{m}$ with $m$ LSH functions, and then encode the points in $\mathcal{Z}^{m}$ space into one-dimensional Zorder values. The closeness of two objects is captured by the length of the longest common prefix between the corresponding Z-order values. C2LSH [8] proposes to use a set of single LSH functions to construct dynamic compound hash functions, where the hash values for each LSH function can be indexed by one-dimensional indexes, such as B-Trees. It uses the collision count among the base LSH functions to estimate the probability of an object being the nearest neighbor of the query $\boldsymbol{q}$. Formally, if the number of LSH functions under which an object colliding with the query $\boldsymbol{q}$ exceeds a certain threshold, it is likely to be the nearest neighbor of $\boldsymbol{q}$ and is selected as a candidate. When expanding the search radius, C2LSH can gradually locate the objects that are farther away from the query $\boldsymbol{q}$ in each LSH function, just like performing range scans on the B-tree. QALSH [9] improves the accuracy of C2LSH by adopting a query-aware bucket partition strategy. Specifically, it uses the query projection as the "anchor" for bucket partition, which avoids partitioning objects closer to the query into different buckets. SRS [10] projects high-dimensional data objects in $\mathbb{R}^{d}$ into a low-dimensional space $\mathbb{R}^{\ell}(\ell<d)$ for exact NN search, 
based on the observation that $\frac{\Delta_{\ell}(\boldsymbol{o}, \boldsymbol{q})}{\operatorname{dist}(\boldsymbol{o}, \boldsymbol{q})}$ follows the standard $\chi^{2}(\ell)$ distribution, where $\Delta_{\ell}(\cdot, \cdot)$ is the distance in the projected space. Given any threshold of the projected distance, the probability of $\left.\Delta_{\ell}(\boldsymbol{o}, \boldsymbol{q})\right)$ not exceeding the threshold can be exactly computed.

Existing studies also consider metrics other than the Euclidean distance. LazyLSH [17] uses a single base index to support multiple $l_{p}$ metrics. FALCONN [13] is proposed as a practical and efficient LSH framework for the angular distance. Concomitant LSH [14] uses concomitant rank order statistics to form the hash functions for cosine similarity. Recently, there are LSH variants trying to improve the I/O efficiency at the expense of quality guarantees, including Entropy-LSH [18], Multi-Probe LSH [7], SK-LSH [19], etc.

There are also many studies focusing on learning based hash methods [3, $5,6,20,21]$. They usually require a rather time-consuming learning process and the learned hash functions are highly data-dependent. Together, it makes them impractical for high-dimensional streaming data.

\section{The PDA-LSH Framework}

In this section, we describe the framework of PDA-LSH indexing and searching. We focus on the design of the index and the search algorithm and leave the detailed internal parameter computation and analysis in Section 4.

\subsection{The Indexing Strategy}

Like QALSH, we use a set $\mathcal{H}$ of query-aware LSH functions to facilitate multiradius search and query-aware bucket partitioning. Formally, functions in $\mathcal{H}$ are in the form of $h_{\boldsymbol{a}}(\boldsymbol{o})=\boldsymbol{a}^{\top} \boldsymbol{o}$, where the entries of $\boldsymbol{a}$ are independent random samples from the standard normal distribution $\mathcal{N}(0,1)$, projecting $\boldsymbol{o} \in \mathcal{D}$ onto the real axis $\mathbb{R}$. Given a query $\boldsymbol{q}$ and a fixed bucket length $w, \mathbb{R}$ is partitioned into consecutive length- $w$ buckets in accordance with $\left[h_{\boldsymbol{a}}(\boldsymbol{q})-\frac{w}{2}, h_{\boldsymbol{a}}(\boldsymbol{q})+\frac{w}{2}\right]$. The PDA-LSH indexing procedure is, for each $h \in \mathcal{H}$, to organize the set of projected values $\{(h(\boldsymbol{o}), \boldsymbol{o}) \mid \boldsymbol{o} \in \mathcal{D}\}$ in an LSM-tree.

With the cascading update mechanism of LSM-trees, the amortized I/O cost for each update is $O\left(\frac{T|\mathcal{H}|}{B} \log |\mathcal{D}|\right)$, where $T$ is the logarithmic size ratio of the LSM-tree and $B$ is the capacity of a disk page [22]. It is worth noting that, in contrast to our choice, QALSH uses B-trees to organize the projected values. Updating the B-trees requires $|\mathcal{H}|$ root-to-leaf traversals, accessing $O(|\mathcal{H}| \log |\mathcal{D}|)$ disk pages. As the value of $B$ is usually much larger than $T$, the index maintenance cost of PDA-LSH is far less than that of QALSH.

\section{2 -Approximate Nearest Neighbor Search}

Besides the conventinal consideration on collisions, the key insight of the $c$-ANN search with PDA-LSH is to use the precise projected distances to 
estimate the distance in the original space. PDA-LSH adopts a thresholdbased strategy: Given pre-specified collision threshold $L$ and projected distance threshold $\Delta$, if a data object $\boldsymbol{o}$ collides with the query $\boldsymbol{q}$ under at least $L \mathrm{LSH}$ functions and the sum of the $L$ projected distances is at most $\Delta$, then $\boldsymbol{o}$ is a good candidate for the $c$-ANN of $\boldsymbol{q}$.

The search is done by solving a series of $(R, c)$-NN problems with gradually expanding radius $R$. During this process, the constraint of $\Delta$ is technically implemented as a constraint on the sum of the squared projected distances (SSPD), which is set proportional to the squared radius, i.e., $\Delta \stackrel{\text { def }}{=} \sqrt{\tau R^{2}}$. Due to their equivalence, we shall proceed our discussion with the parameter $\tau$ instead of $\Delta$ hereafter.

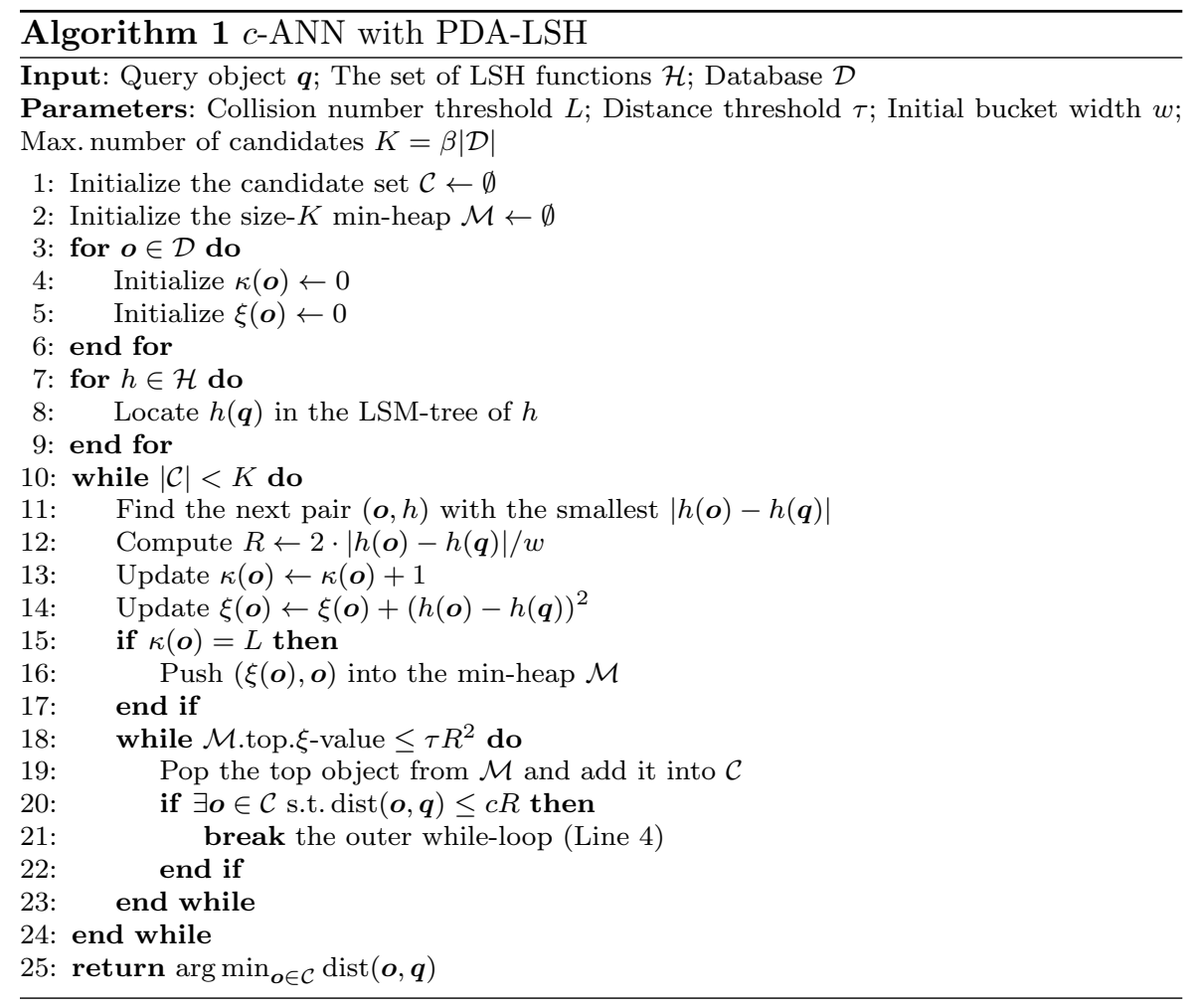

Algorithm 1 presents the $c$-ANN search with PDA-LSH. The parameters $\tau$ and $w$ are specified for an initial radius of $R=1$. Given a query $\boldsymbol{q}$, the algorithm first computes its projections and locate them in the LSM-trees (Lines 7-9). Each projection $h(\boldsymbol{q})$ is used as an "anchor" for bucket partitioning in all levels of the LSM-tree. The algorithm then expands the bucket boundary (i.e., the search radius $R$ ) adaptively by accessing objects in ascending order of their projected distances to the anchors (Line 11). As the initial bucket width $w$ is for $R=1$, it is trivial to derive the next radius with the observed 


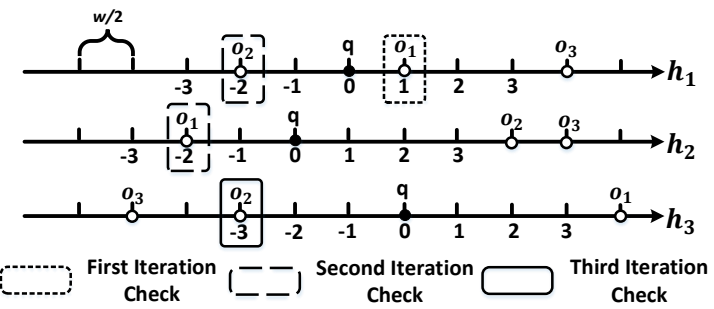

Fig. 1 Running example of algorithm 1

projected distance (Line 12). For each object $\boldsymbol{o}$ appearing within the radius $R$, the algorithm updates its collisions and SSPD (Lines 13-14). Once an object $\boldsymbol{o}$ is found sufficient collisions with the query, it is pushed into a min-heap $\mathcal{M}$ with its SSPD for further investigation (Lines 15-17). Objects in the heap with SSPDs at most $\tau R^{2}$ are considered as good candidates (Lines 18-23). Finally, the algorithm terminates (Lines 20-22) if one of the following two conditions holds:

1. There exists $\boldsymbol{o} \in \mathcal{C}$ with $\operatorname{dist}(\boldsymbol{o}, \boldsymbol{q}) \leq c R$ under the current search radius $R$; or

2. $K=\beta|\mathcal{D}|$ candidates have already been found. ${ }^{1}$

Example. Figure 1 shows a running example of Algorithm 1. Assume that the approximation ratio $c=2$, the collision threshold $L=2$, and the distance threshold $\tau=0.2 w^{2}$, where $w$ is the initial bucket width. Figure 1 shows the relative positions of three objects, $\boldsymbol{o}_{1}, \boldsymbol{o}_{2}$, and $\boldsymbol{o}_{3}$, under three LSH functions, $h_{1}, h_{2}$, and $h_{3}$, with respect to the query $\boldsymbol{q}$. PDA-LSH checks each object in ascending order of their projected distances to $\boldsymbol{q}$. In the first iteration, $\left|h_{1}\left(\boldsymbol{o}_{1}\right)-h_{1}(\boldsymbol{q})\right|=0.5 w$ is the minimum projected distance, thus the radius is updated as $R=(2)(0.5 w) / w=1$. Then, PDA-LSH updates the collision and projected distance information for object $\boldsymbol{o}_{1}$, i.e., $\kappa\left(\boldsymbol{o}_{1}\right)=1$ and $\xi\left(\boldsymbol{o}_{1}\right)=\left|h_{1}\left(\boldsymbol{o}_{1}\right)-h_{1}(\boldsymbol{q})\right|^{2}=0.25 w^{2}$. As $\kappa\left(\boldsymbol{o}_{1}\right)<L, \boldsymbol{o}_{1}$ is not yet considered a candidate and the termination conditions of Algorithm 1 do not yet hold, hence the algorithm continues. In the second iteration, $\left(\boldsymbol{o}_{2}, h_{1}\right)$ and $\left(\boldsymbol{o}_{1}, h_{2}\right)$ are found due to $\left|h_{1}\left(\boldsymbol{o}_{2}\right)-h_{1}(\boldsymbol{q})\right|=\left|h_{2}\left(\boldsymbol{o}_{1}\right)-h_{2}(\boldsymbol{q})\right|=w$. Algorithm 1 thus updates $R=\frac{2}{w} \cdot w=2, \kappa\left(\boldsymbol{o}_{1}\right)=2, \xi\left(\boldsymbol{o}_{1}\right)=1.25 w^{2}, \kappa\left(\boldsymbol{o}_{2}\right)=1$, and $\xi\left(\boldsymbol{o}_{2}\right)=w^{2}$. As $\kappa\left(\boldsymbol{o}_{1}\right)$ reaches $L=2$, the pair $\left(1.25 w^{2}, \boldsymbol{o}_{1}\right)$ is pushed into the heap. However, since $\xi\left(\boldsymbol{o}_{1}\right)=1.25 w^{2}>0.8 w^{2}=\tau R^{2}, \boldsymbol{o}_{1}$ is still not a candidate. Again, the algorithm continues with $\boldsymbol{o}_{1}$ in the heap. In the third iteration, $\left(\boldsymbol{o}_{2}, h_{3}\right)$ is found, and the algorithm updates $R=3, \kappa\left(\boldsymbol{o}_{2}\right)=2$ and $\xi\left(\boldsymbol{o}_{2}\right)=3.25 w^{2}$, increased by $\left|h_{3}\left(\boldsymbol{o}_{2}\right)-h_{3}(\boldsymbol{q})\right|^{2}=2.25 w^{2}$. The pair $\left(3.25 w^{2}, \boldsymbol{o}_{2}\right)$ is pushed into the heap as $\kappa\left(\boldsymbol{o}_{2}\right)$ reaches the threshold $L=2$. Moreover, after updating $R$, it now holds that $\xi\left(\boldsymbol{o}_{1}\right)=1.25 w^{2}<1.8 w^{2}=\tau R^{2}$. Thus, $\boldsymbol{o}_{1}$ is a candidate and the Euclidean distance between $\boldsymbol{o}_{1}$ and $\boldsymbol{q}$ is computed. Then, it is discovered that

\footnotetext{
1 Note that Algorithm 1 sets $K$ proportional to the total number of objects, $|\mathcal{D}|$. The ratio, denoted $\beta$, controls the false positive rate during the search.
} 
$\operatorname{dist}\left(\boldsymbol{o}_{1}, \boldsymbol{q}\right)=4<6=c R$, satisfying the termination condition of Algorithm 1 . Therefore, Algorithm 1 returns $\boldsymbol{o}_{1}$ as a result.

$\boldsymbol{c}$ - $\boldsymbol{k}$-ANN extension. Algorithm 1 can be easily extended to support the $c-k$-ANN search by changing the termination conditions into:

1. There exist $k$ objects in $\mathcal{C}$ whose distance to the query $\boldsymbol{q}$ are at most $c R$; or

2. $\beta|\mathcal{D}|+k-1$ candidates have already been found.

In either case, the $k$ nearest objects from $\mathcal{C}$ are the $c$ - $k$-ANN results.

Advantages. Compared with QALSH [9], which implicitly considers the projected distances via collisions, PDA-LSH explicitly utilizes the projected distances to further distinguish objects hashed into the same bucket. With carefully chosen parameters $L$ and $\tau$, PDA-LSH can achieve the same quality guarantees with a lower collision threshold than that of QALSH. As a result, the research cost is effectively reduced. Also, compared with SRS [10], PDA-LSH makes use of more projections to achieve more accurate distance estimations, thereby yielding better result quality.

Practical Implementation. In order to inspect objects in ascending order of their projection distances to the query object, a specific heap storing the next objects of each LSM-tree has to be maintained, which will result in high overhead of runtime. As a matter of fact, the purpose of this data access strategy is to expand the search radius incrementally. To improve the efficiency of our search scheme, we propose a practical implementation, which eliminates the cost of heap maintenance and tries to attain the goal of incremental bucket width expansion. We enlarge the search radius linearly with a growth rate of a certain proportion of $\bar{s}$, where $\bar{s}$ is the average distance between objects in the database.

\section{Parameter Settings}

In this section, we illustrate how internal parameters are computed to ensure the correctness of PDA-LSH, i.e., how Algorithm 1 returns true $c$-ANN results with at least constant success probability. Previous study [23] has shown that, if objects are accessed in ascending order of their projected distances, the correctness of $(R, c)$-NN implies the correctness of $c$-ANN. Therefore, we focus our discussion on $(R, c)-\mathrm{NN}$.

\subsection{Success Probability of PDA-LSH for $(R, c)-\mathrm{NN}$}

PDA-LSH directly solves the $(R, c)$-NN problem by imposing query-aware buckets with width $w R$. That is, PDA-LSH locates objects falling within the query-anchored bucket $\left[h(\boldsymbol{q})-\frac{w R}{2}, h(\boldsymbol{q})+\frac{w R}{2}\right]$. To ensure the correctness of Algorithm 1, the following two properties must hold at the same time with constant probability. 
- Property $\mathbf{P}_{1}$ : If there exists an object $\boldsymbol{o}$ such that $\operatorname{dist}(\boldsymbol{o}, \boldsymbol{q}) \leq R$, then $\boldsymbol{o}$ is added into the candidate set $\mathcal{C}$.

- Property $\mathbf{P}_{2}$ : The total number of false positives is no more than $K$, where a false positive is an $\boldsymbol{o} \in \mathcal{C}$ with $\operatorname{dist}(\boldsymbol{o}, \boldsymbol{q})>c R$.

To further compute the probability $\operatorname{Pr}\left[\mathbf{P}_{1} \cap \mathbf{P}_{2}\right]$, our discussion will intensively use two important functions, $\operatorname{Col}_{\boldsymbol{q}}(\boldsymbol{o}, R)$ and $\operatorname{SSPD}_{\boldsymbol{q}}(\boldsymbol{o}, j)$, defined as follows.

Definition 2 Define $\mathrm{Col}_{\boldsymbol{q}}(\boldsymbol{o}, R)$ as the number of collisions within radius $R$, i.e., the number of LSH functions $h \in \mathcal{H}$ such that $h(\boldsymbol{o}) \in$ $\left[h(\boldsymbol{q})-\frac{w R}{2}, h(\boldsymbol{q})+\frac{w R}{2}\right]$. Also, define $\operatorname{SSPD}_{\boldsymbol{q}}(\boldsymbol{o}, j)$ as the sum of the $j$ smallest squared projected distances between $\boldsymbol{o}$ and $\boldsymbol{q}$. When there is no ambiguity on the query object $\boldsymbol{q}$, we write the functions as $\operatorname{Col}(\boldsymbol{o}, R)$ and $\operatorname{SSPD}(\boldsymbol{o}, j)$, omitting the subscript $\boldsymbol{q}$.

Let $s$ be the variable for the object-query distance, i.e., $s=\operatorname{dist}(\boldsymbol{o}, \boldsymbol{q})$. We consider the following two parameterized probabilistic events:

- $\mathbf{E}_{1}(s, R): \operatorname{Col}(\boldsymbol{o}, R) \geq L$.

- $\mathbf{E}_{2}(s, R): \operatorname{SSPD}(\boldsymbol{o}, L) \leq \tau R^{2}$.

In addition, let the event $\mathbf{E}_{0}(s, R) \stackrel{\text { def }}{=} \mathbf{E}_{1}(s, R) \cap \mathbf{E}_{2}(s, R)$. We have the following main theorem.

Theorem 1 If the collision and distance thresholds $L$ and $\tau$ are chosen such that (1) $\operatorname{Pr}\left[\boldsymbol{E}_{0}(s, R) \mid s \leq R\right] \geq 1-\delta$ and (2) $\operatorname{Pr}\left[\boldsymbol{E}_{0}(s, R) \mid s>c R\right]<\frac{\beta}{2}$, then $\operatorname{Pr}\left[\boldsymbol{P}_{1} \cap \boldsymbol{P}_{2}\right] \geq \frac{1}{2}-\delta$.

Proof According to Line 9 of Algorithm 1, it is easy to see that

$$
\operatorname{Pr}\left[\mathbf{P}_{1}\right]=\operatorname{Pr}\left[\mathbf{E}_{0}(s, R) \mid s \leq R\right] \geq 1-\delta .
$$

Next, consider the set of false positives,

$$
S=\left\{\boldsymbol{o} \in \mathcal{D} \mid \mathbf{E}_{0}(s, R) \wedge \operatorname{dist}(\boldsymbol{o}, \boldsymbol{q})>c R\right\} .
$$

Since there are at most $|\mathcal{D}|$ objects whose distances to $\boldsymbol{q}$ are larger than $c R$, by applying Markov's Inequality, we obtain

$$
\operatorname{Pr}[|S| \geq \beta|\mathcal{D}|] \leq \frac{\mathbb{E}[|S|]}{\beta|\mathcal{D}|}<\frac{\frac{\beta}{2}|\mathcal{D}|}{\beta|\mathcal{D}|}=\frac{1}{2} .
$$

Thus, $\operatorname{Pr}\left[\mathbf{P}_{2}\right]=\operatorname{Pr}[|S|<\beta|\mathcal{D}|]>\frac{1}{2}$. Taken together, we have $\operatorname{Pr}\left[\mathbf{P}_{2} \cap \mathbf{P}_{2}\right]=$ $\operatorname{Pr}\left[\mathbf{P}_{1}\right]+\operatorname{Pr}\left[\mathbf{P}_{2}\right]-\operatorname{Pr}\left[\mathbf{P}_{1} \cup \mathbf{P}_{2}\right] \geq \frac{1}{2}-\delta$.

Theorem 1 implies the correctness of PDA-LSH for $(R, c)$-NN. Given userspecified inputs $c, \beta$, and $\delta$, the task now is to choose proper thresholds $L$ and $\tau$ to meet the prerequisites of Theorem 1 . The key is to compute the probability

$$
\operatorname{Pr}\left[\mathbf{E}_{0}(s, R)\right]=\operatorname{Pr}\left[\mathbf{E}_{1}(s, R)\right] \cdot \operatorname{Pr}\left[\mathbf{E}_{2}(s, R) \mid \mathbf{E}_{1}(s, R)\right] .
$$


Computing the first term, $\operatorname{Pr}\left[\mathbf{E}_{1}(s, R)\right]$, is relatively easy. For any object $\boldsymbol{o}$ with $\operatorname{dist}(\boldsymbol{o}, \boldsymbol{q})=s$, let $p(s, R)$ denote the collision probability under a random $h \in \mathcal{H}$ with bucket width $w R$. It has been shown that $h(\boldsymbol{o})-h(\boldsymbol{q})$ observes the normal distribution $\mathcal{N}\left(0, s^{2}\right)[18]$. Hence,

$$
p(s, R)=\operatorname{Pr}\left[|h(\boldsymbol{o})-h(\boldsymbol{q})| \leq \frac{w R}{2}\right]=2 \Phi\left(\frac{w R}{2 s}\right)-1,
$$

where $\Phi(\cdot)$ is the CDF of $\mathcal{N}(0,1)$. Then,

$$
\operatorname{Pr}\left[\mathbf{E}_{1}(s, R)\right]=\sum_{i=L}^{|\mathcal{H}|}\left(\begin{array}{c}
|\mathcal{H}| \\
i
\end{array}\right) p(s, R)^{i}(1-p(s, R))^{|\mathcal{H}|-i} .
$$

Computing the second term, $\operatorname{Pr}\left[\mathbf{E}_{2}(s, R) \mid \mathbf{E}_{1}(s, R)\right]$, however, is challenging as there exsits no closed-form formula. The exact computation is prohibitive. Therefore, we resort to a heuristic and practical method, which consists of two steps. First, it determines the smallest size of $\mathcal{H}$ while securing the tenability of Theorem 1 (i.e., with $\mathcal{H}$, there exists $L$ and $\tau$ satisfying the prerequisites of Theorem 1). Then, it finds a parameter setting $(L, \tau)$ with minimum $L$. The intuition is that, with minimized $\mathcal{H}$ and $L$, Algorithm 1 may find the candidates as early as possible at the least possible cost.

\subsection{Determining the Number of LSH Functions}

Given the search radius $R$ and the distance $s=\operatorname{dist}(\boldsymbol{o}, \boldsymbol{q})$, the number of collisions under $m$ LSH functions observes the binomial distribution $\mathcal{B}(m, p(s, R))$, and the probability of $\boldsymbol{o}$ and $\boldsymbol{q}$ colliding more than some given threshold is exactly the right-tail probability of the distribution. To estimate the right-tail probability, instead of directly working with $\mathcal{B}(m, p(s, R))$, we propose to approximate $\mathcal{B}(m, p(s, R))$ by the normal distribution $\mathcal{N}\left(m \cdot p(s, R), m \cdot q^{2}(s, R)\right)$, where $q(s, R) \stackrel{\text { def }}{=} \sqrt{p(s, R)(1-p(s, R))}$. The benefit is that estimating the right-tail probability of a normal distribution will result in a much smaller $m$. Specifically, Theorem 1 requires that

$$
\Phi\left(\frac{L-m \cdot p(R, R)}{\sqrt{m} \cdot q(R, R)}\right) \leq \delta
$$

and

$$
\Phi\left(\frac{L-m \cdot p(c R, R)}{\sqrt{m} \cdot q(c R, R)}\right)>1-\frac{\beta}{2}
$$

bounding $L$ from above and below, respectively. It can be calculated that, in order to secure a valid lower and upper bounding of $L$, the smallest value $m$ may take is

$$
m=\left\lceil\left(\frac{\Phi^{-1}\left(1-\frac{\beta}{2}\right) q(c R, R)-\Phi^{-1}(\delta) q(R, R)}{p(R, R)-p(c R, R)}\right)^{2}\right\rceil .
$$


4.3 Determining the Collision and Distance Thresholds

Once $m$ is determined, the goal is to choose a proper set of $L$ and $\tau$. Although one can solve an $L$ from the above bounding conditions associated with the normal estimation, the result could be unsatisfactory as the above process does not take advantage of the exact projected distances. Alternatively, we consider using a Monte Carlo method to estimate the conditional probability $\operatorname{Pr}\left[\mathbf{E}_{2}(s, R) \mid \mathbf{E}_{1}(s, R)\right]$ in Equation 1. The following two technical lemmata are useful.

Lemma 1 (Monotonicity) For arbitrary $\boldsymbol{o}_{1}, \boldsymbol{o}_{2}, \boldsymbol{q} \in \mathbb{R}^{d}$, let $s_{1}=\operatorname{dist}\left(\boldsymbol{o}_{1}, \boldsymbol{q}\right)$ and $s_{2}=\operatorname{dist}\left(\boldsymbol{o}_{2}, \boldsymbol{q}\right)$. If $s_{1}<s_{2}$, then

(1) $\operatorname{Pr}\left[\boldsymbol{E}_{1}\left(s_{1}, R\right)\right]>\operatorname{Pr}\left[\boldsymbol{E}_{1}\left(s_{2}, R\right)\right]$,

(2) $\operatorname{Pr}\left[\boldsymbol{E}_{2}\left(s_{1}, R\right) \mid \boldsymbol{E}_{1}\left(s_{1}, R\right)\right]>\operatorname{Pr}\left[\boldsymbol{E}_{2}\left(s_{2}, R\right) \mid \boldsymbol{E}_{1}\left(s_{2}, R\right)\right]$, and

(3) $\operatorname{Pr}\left[\boldsymbol{E}_{0}\left(s_{1}, R\right)\right]>\operatorname{Pr}\left[\boldsymbol{E}_{0}\left(s_{2}, R\right)\right]$.

Proof We put the proof in Appendix A so that it does not distract the main discussion.

Lemma 2 (Cancellation) For any $c, s, R>0$, it holds that $\operatorname{Pr}\left[\boldsymbol{E}_{0}(c s, c R)\right]=$ $\operatorname{Pr}\left[\boldsymbol{E}_{0}(s, R)\right]$.

Proof Trivial via a scaling in the Euclidean space.

The lemmata indicate the following result.

Theorem $2 A$ threshold setting $(L, \tau)$ is feasible if it satisfies (1) $\operatorname{Pr}\left[\boldsymbol{E}_{0}(1,1)\right] \geq 1-\delta$, and (2) $\operatorname{Pr}\left[\boldsymbol{E}_{0}(c, 1)\right]<\frac{\beta}{2}$.

Proof Lemma 1 implies that it suffices to consider two critical conditions, $\operatorname{Pr}\left[\mathbf{E}_{0}(R, R)\right] \geq 1-\delta$ and $\operatorname{Pr}\left[\mathbf{E}_{0}(c R, R)\right]<\frac{\beta}{2}$. Then, Lemma 2 further reduces the left-hand side probabilities to $\operatorname{Pr}\left[\mathbf{E}_{0}(1,1)\right]$ and $\operatorname{Pr}\left[\mathbf{E}_{0}(c, 1)\right]$.

Based on Theorem 2, we first show how to estimate the probability $\operatorname{Pr}\left[\mathrm{E}_{0}(s, 1)\right]$ with respect to specific $L$ and $\tau$, and then present an algorithm to find plausible $L$ and $\tau$.

The Monte Carlo estimation of $\operatorname{Pr}\left[\mathrm{E}_{0}(s, 1)\right]$ is to take $N$ samples, $\boldsymbol{o}_{1}, \boldsymbol{o}_{2}, \cdots, \boldsymbol{o}_{N}$, uniformly at random on the sphere $S^{d-1}(s)=$ $\left\{\boldsymbol{o} \in \mathbb{R}^{d}:\|\boldsymbol{o}\|=s\right\}$; this is to simulate objects of distance $s$ to the query (i.e., the origin). Then, given $L$ and $\tau$, we count the number of $\boldsymbol{o}_{i}$ 's satisfying (1) $\operatorname{Col}\left(\boldsymbol{o}_{i}, 1\right) \geq L$ and $(2) \operatorname{SSPD}\left(\boldsymbol{o}_{i}, L\right) \leq \tau$. If there are $N^{\prime}$ such samples, then $\operatorname{Pr}\left[\mathrm{E}_{0}(s, 1)\right] \approx \frac{N^{\prime}}{N}$ when $N$ is sufficiently large.

Now, assume that we have a set, $D_{s}$, of $N$ random samples from the sphere $S^{d-1}(s) \subset \mathbb{R}^{d}$. Consider the multisets ${ }^{2}$

$$
A_{s, j} \stackrel{\text { def }}{=}\left\{\operatorname{SSPD}(\boldsymbol{o}, j): \boldsymbol{o} \in D_{s}, \operatorname{Col}(\boldsymbol{o}, 1) \geq j\right\}
$$

\footnotetext{
2 That is, SSPD values of different data objects are considered distinct elements in $A_{s, j}$ even though they might be equal. Thus, the size of the multiset $A_{s, j}$ is precisely the number of objects colliding at least $j$ times with the query (within radius $R=1$ ).
} 
for $s=1, c$ and $j=1,2, \cdots, m$. If for some $j$, the $(1-\delta) N$-th smallest value, $v$, in $A_{1, j}$ can bound at most $\frac{\beta}{2} N-1$ values in $A_{c, j}$ from above, then according to the Monte Carlo process, $(L, \tau)=(j, v)$ satisfies both $\operatorname{Pr}\left[\mathbf{E}_{0}(1,1)\right] \geq 1-\delta$ and $\operatorname{Pr}\left[\mathbf{E}_{0}(c, 1)\right]<\frac{\beta}{2}$. In other words, according to Theorem $2,(L, \tau)=(j, v)$ is a feasible threshold setting. The following Algorithm 2 implements exactly this idea.

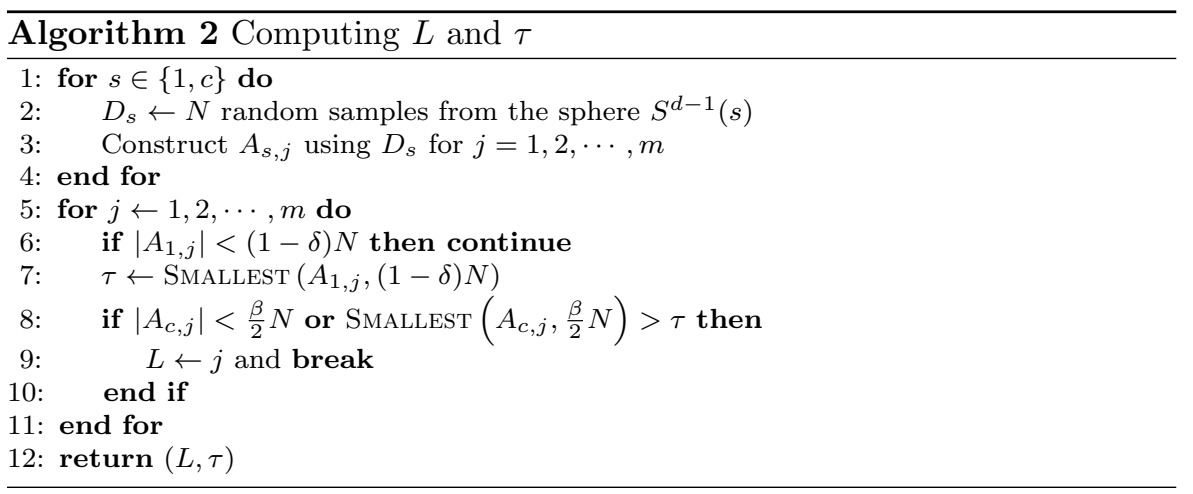

Algorithm 2 searches for the smallest feasible $L$ by trying $L=1,2, \cdots$ in order. The procedure relies only on user-specified input parameters $(c, \beta$, and $\delta$ ) and $m$, the number of LSH functions determined in Section 4.2.

\section{Experiments}

In this section, we evaluate the efficiency and accuracy of our proposed method, PDA-LSH. We mainly compare PDA-LSH with QALSH [9] and SRS [10], which are two state-of-the-art I/O efficient algorithms with theoretical guarantees on the result quality.

\subsection{Setup}

Environment. The experiments were ran on a workstation powered by Intel Xeon Gold-6148 CPU on Linux (Ubuntu 16.04), having a 15K RPM disk. All the experiments were conducted using the direct I/O mode to eliminate influences caused by the data caching of file systems.

Datasets. We experimented on four real-world datasets. The characteristics of the datasets are summarized as follows:

- Sift $^{3}$ consists of one million 128-dimensional SIFT feature vectors from the ANN_SIFT1M dataset. The dataset is extracted from the INRIA Holidays images.

\footnotetext{
3 http://corpus-texmex.irisa.fr/
} 
- Msong ${ }^{4}$ contains 994,020 audio feature vectors of 420 dimensions, which are extracted from one million western popular music pieces.

- Gist ${ }^{5}$ is an image dataset which contains one million 960-dimensional data points. The dataset is extracted from a combination of the Holiday image dataset and the Flickr1M dataset [24].

- Trevi $^{6}$ consists of 100,900 feature vectors, each of 4,096-dimensional, extracted from image patches are taken from the Photo Tourism reconstructions of Trevi Fountain.

Table 1 Statistics of Datasets

\begin{tabular}{|c|c|c|}
\hline Dataset & Dimensionality $d$ & Size $|\mathcal{D}|$ \\
\hline Sift & 128 & $1,000,000$ \\
\hline Msong & 420 & 994,020 \\
\hline Gist & 960 & $1,000,000$ \\
\hline Trevi & 4,096 & 100,900 \\
\hline
\end{tabular}

Table 1 summarizes the statistics of the datasets. To simulate streaming data, in each dataset, we sample $5 \%$ of the data objects as updates, which can reflect real-world distributions. The query set consists of 100 queries. For Sift and Gist, queries are sampled from their test sets. For Msong and Trevi, queries are sampled from the data objects.

Parameter settings. We set the page size to $8 \mathrm{~KB}$. We use 4-byte integers to store the object IDs and 4-byte floats to store the hash projections. To be fair, the success probability of all algorithms is set to $\frac{1}{2}-\frac{1}{e}$, which is the default value of most LSH-based methods. The default approximate ratio $c$ is set to 2 , which provides a nice trade-off between query accuracy and efficiency. Following QALSH, for each dataset $\mathcal{D}$, we set the false positive percentage $\beta=\frac{100}{|\mathcal{D}|}$ (i.e., the maximum number of candidates $K=100$ ) to restrict the number of random I/Os resulting from candidates verification. For SRS, the number of projections is set to 6. Both PDA-LSH and QALSH use the bucket width $w=2.719$ so that the gap between $p(R, R)$ and $p(c R, R)$ (see Equation 2 ) is maximized. For the Monte Carlo estimation process used in PDA-LSH, we set the number of random samples, $N$, to 100 million (see Algorithm 2).

\subsection{Evaluation on Space Consumption}

To evaluate the space cost of the algorithms, we list the number of required LSH projections and the index sizes of the algorithms in Table 2. We see that the index sizes of these methods are independent of the data dimensionality $d$. Indeed, LSH methods only store projections in the index and the number of

\footnotetext{
4 http://www.ifs.tuwien.ac.at/mir/msd/

5 http://corpus-texmex.irisa.fr/

6 http://phototour.cs.washington.edu/patches/
} 
required LSH projections is irrelevant to $d$. SRS has the smallest size because it uses much fewer projections than the other methods. The index size of PDALSH is 2.6-3 times smaller than QALSH. There are two primary reasons. One is that PDA-LSH uses fewer separate indexes as it derives a more accurate estimation of the number of required LSH functions (see Section 4.2). The other reason is that LSM-tree used by PDA-LSH is more space-efficient than B-tree used by QALSH when handling updates. For example, on Gist dataset, the space usage of B-tree is roughly $71 \%$ (i.e., $\frac{83 \times 10^{6} \times 8 \text { Byte }}{891.88 \mathrm{MB}} \approx 0.71$ ), while LSM-tree has nearly $100 \%$ usage.

Table 2 Internal Parameters and Index Sizes (MB) of PDA-LSH, QALSH, and SRS

\begin{tabular}{|c|c|c|c|c|c|c|c|c|c|}
\hline \multirow{2}{*}{ Dataset } & \multicolumn{3}{|c|}{ PDA-LSH } & \multicolumn{3}{|c|}{ QALSH } & \multicolumn{3}{|c|}{ SRS } \\
\cline { 2 - 10 } & $|\mathcal{H}|$ & $L\left(L^{\prime}\right)$ & Size & $|\mathcal{H}|$ & $L$ & Size & $|\mathcal{H}|$ & $L$ & Size \\
\hline Sift & 45 & $33(36)$ & 346.09 & 83 & 63 & 905.92 & 6 & - & 38.7 \\
\hline Msong & 45 & $33(36)$ & 343.32 & 83 & 63 & 886.54 & 6 & - & 37.6 \\
\hline Gist & 45 & $33(36)$ & 346.09 & 83 & 63 & 891.88 & 6 & - & 37.9 \\
\hline Trevi & 32 & $24(26)$ & 25.14 & 68 & 51 & 74.78 & 6 & - & 4.3 \\
\hline
\end{tabular}

\subsection{Evaluation on Update Performance}

In this section, we compare the update performance of PDA-LSH with existing methods. We report the average elapsed time per update. The results are shown in Figure 2. We can see that PDA-LSH always achieves the best per-

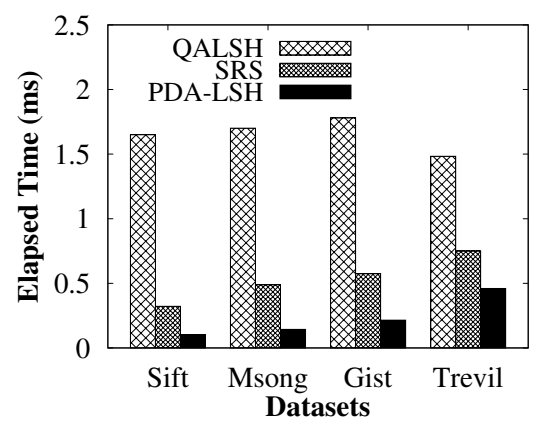

Fig. 2 Update performance comparison.

formance and is up to 15.8 times more efficient than the existing methods. For example, on Sift dataset, the average response time per update for PDA-LSH, QALSH, and SRS are $0.104 \mathrm{~ms}, 1.65 \mathrm{~ms}$, and $0.322 \mathrm{~ms}$, respectively. This is because the LSM-trees bring a significant benefit in terms of write efficiency. Note that the update for SRS is relatively inefficient although it maintains a 
single R-tree. R-tree is inherently not suitable for updates, since an update might modify multiple nodes due to the adjustments of overlapping MBRs.

\subsection{Evaluation on Search Performance}

In this section, we evaluate the search performance of the three algorithms. Following previous studies $[9,10]$, we adopt three metrics in our search performance evaluations: overall ratio, I/O cost and running time. For $c-k-\mathrm{NN}$ search, the overall ratio is defined as

$$
\frac{1}{k} \sum_{i=1}^{k} \frac{\operatorname{dist}\left(\boldsymbol{o}_{i}, \boldsymbol{q}\right)}{\operatorname{dist}\left(\boldsymbol{o}_{i}^{*}, \boldsymbol{q}\right)}
$$

where $\boldsymbol{o}_{i}$ is the $i$-th returned object and $\boldsymbol{o}_{i}^{*}$ is the true $i$-th nearest neighbor. The I/O cost is defined as the number of disk pages to be accessed, which consists of the cost of index accesses to find candidates and the cost of data accesses for candidate verification. The running time is the measured elapsed time per query. The I/O cost and running time are used to evaluate the efficiency of the three algorithms, and the overall ratio is used to evaluate their accuracy. All of the three metrics are averaged over the performed queries.

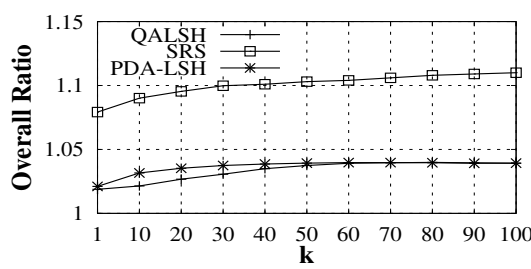

(a) Sift

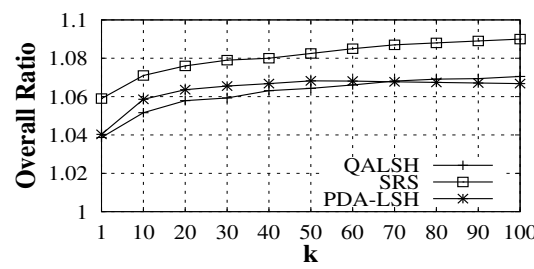

(c) Gist

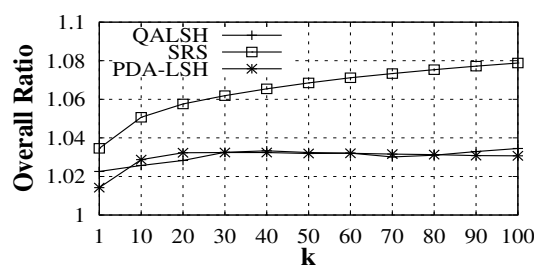

(b) Msong

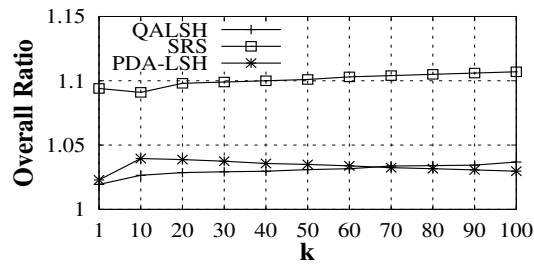

(d) Trevi

Fig. 3 Overall ratio comparison with varying $k$.

Overall ratio. We evaluate the overall ratio of the three algorithms for 2$k$-ANN searches with $k$ varying from 1 to 100 . The results are shown in Figure 3. We observe that both PDA-LSH and QALSH offer much higher accuracy than SRS, especially on datasets with higher dimensions. For example, on Trevi dataset, when $k=1$, the overall ratio for PDA-LSH, QALSH, and SRS are 
1.023, 1.02, and 1.094, respectively. The main reason for this is that SRS cannot scale to more than 6 projections due to practical limitations of R-tree. In fact, when looking over the overall ratios of the performed queries, we observed that some SRS results were particularly high and even exceeded 1.7. In contrast, the underlying one-dimensional indexes make it convenient for PDA-LSH and QALSH to scale to more projections. With abundant projection information, robust quality guarantees (i.e., the upper bounds on the false negative and false positive rates) can be achieved.

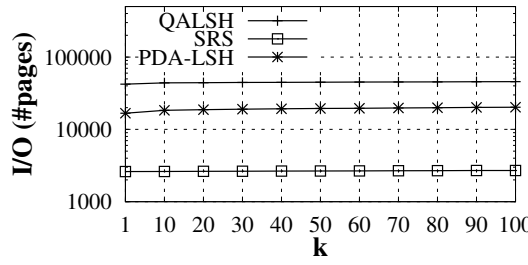

(a) Sift

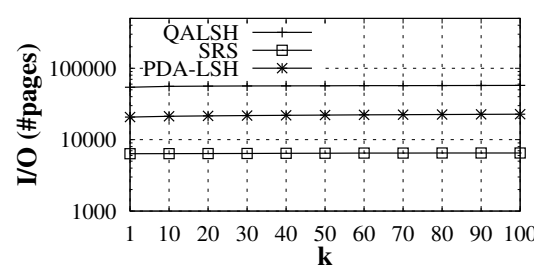

(c) Gist

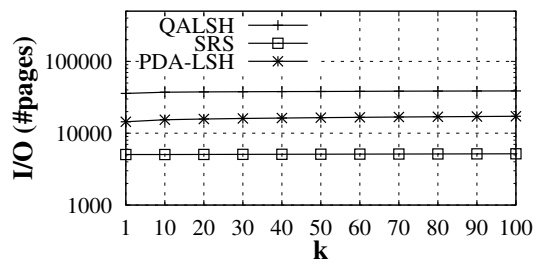

(b) Msong

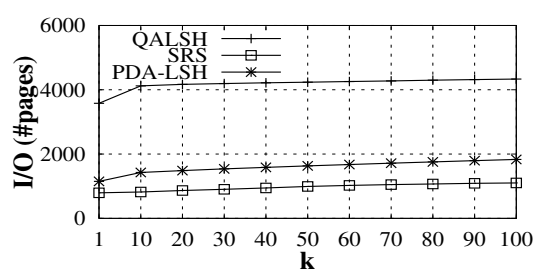

(d) Trevi

Fig. $4 \mathrm{I} / \mathrm{O}$ cost comparison with varying $k$.

I/O cost. We also measure the $\mathrm{I} / \mathrm{O}$ cost of the three algorithms for $2-k$ ANN searches with $k$ varying from 1 to 100. Figure 4 shows the result. We can see that SRS has the smallest I/O cost due to its small index size. However, as described before, this is often at the expense of result accuracy. Note that the gap of I/O cost between SRS and the other algorithms narrows when the data dimensionality $d$ increases. This is because the error of distance estimation increases with $d$ when a fixed number (i.e., 6) of projections are applied. As a result, SRS has to check more objects to meet its termination conditions. We also observe that the I/O cost of PDA-LSH is 2.3-3.1 times less than QALSH while offering comparable result accuracy. This is not only because PDA-LSH has less separate indexes, but also because it uses the precise projected distances to further distinguish objects falling into the same bucket. As Table 2 shows, the threshold $L$ of precise projection based method is smaller than the threshold $L^{\prime}$ (in brackets) derived by our method. With a lower collision threshold, candidates can be found earlier, and as a result, the index scan cost is reduced.

Running time. The overall search performance comparison of the three algorithms is shown in Figure 5. We can see that our approach shows good 


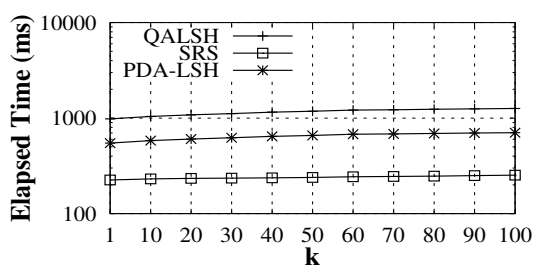

(a) Sift

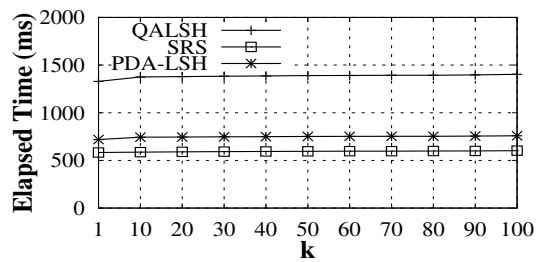

(c) Gist

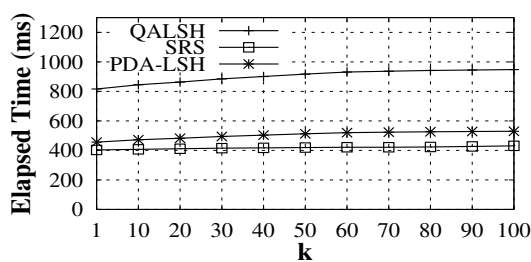

(b) Msong

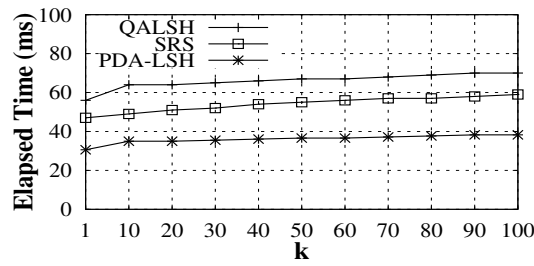

(d) Trevi

Fig. 5 Running time comparison with varying $k$.

scalability with the increasing of data dimensionality, just as we have observed for the I/O cost. More specifically, when the data dimensionality is large $(d \geq$ 420 ), the performance of PDA-LSH is comparable to (or even better than) that of SRS although its I/O cost is higher. For example, on Trevi dataset, when $k=1$, the average response time per query for PDA-LSH, QALSH, and SRS are $30.6 \mathrm{~ms}, 56.5 \mathrm{~ms}$, and $40.3 \mathrm{~ms}$, respectively. The main reason is that PDA-LSH benefits from the fast sequential I/Os offered by LSM-tree, while SRS consumes a large number of poor random I/Os when traversing the Rtree. As expected, PDA-LSH is around 2 times faster than QALSH due to its smaller I/O cost. Note that when compared with QALSH, the improvement of running time is slightly less than that of $\mathrm{I} / \mathrm{O}$ cost.

\subsection{Impact of the Approximation Ratio}

In this section, we investigate the impact of the approximation ratio $c$ on the performance of PDA-LSH. We evaluate PDA-LSH on four real-world datasets using different values of $c$, and report the overall ratio, I/O cost, and running time. Due to the space limit, we only show the results on Msong and Trevi datasets in Figure 6. The results on the other datasets have similar trends.

From Figure 6, we observe that the results on different choice of $c$ exhibit a trade-off between efficiency and accuracy. Specifically, Figure 6(a)-(b) depict the overall ratio of PDA-LSH for different values of $c$. We can see that the overall ratio decreases significantly when $c$ becomes smaller. When $c=1.5$, the overall ratio is barely above 1.0 , which indicates that the accuracy of returned results is especially high. Figure 6(c)-(f) give the I/O cost and running time of PDA-LSH when varying the approximation ratio $c$. In contrast to the result on overall ratio, the I/O cost and running time increase monotonically with the 


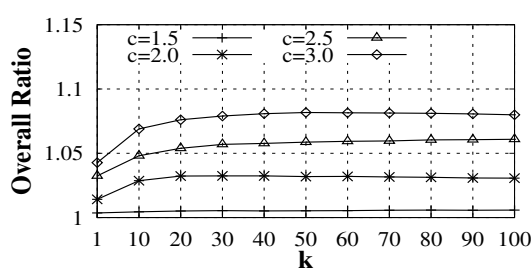

(a) Overall ratio on Msong

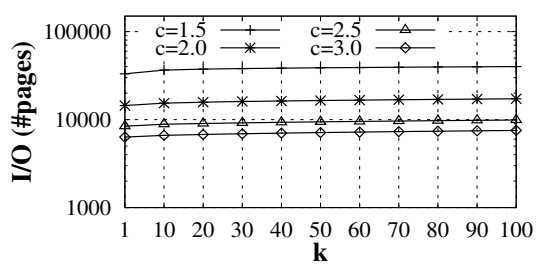

(c) I/O cost on Msong

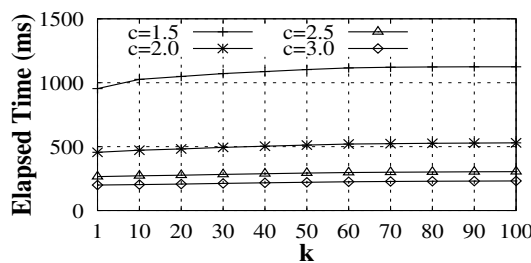

(e) Running time on Msong

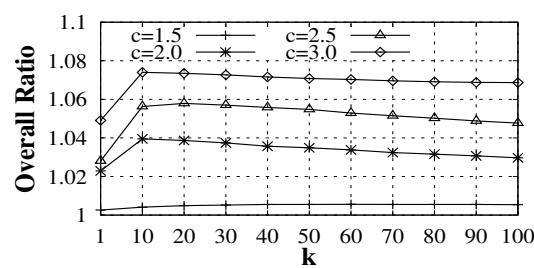

(b) Overall ratio on Trevi

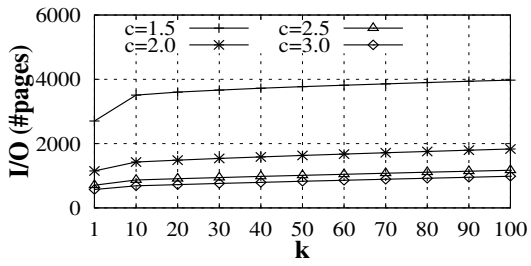

(d) I/O cost on Trevi

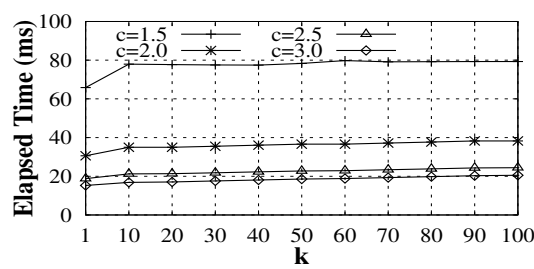

(f) Running time on Trevi

Fig. 6 Performance of PDA-LSH with varying approximation ratio $c$

decrease of $c$. For example, on Trevi dataset, when $k=1$ and $c$ increases from 1.5 to 3.0, the running time of PDA-LSH are $65 \mathrm{~ms}, 30 \mathrm{~ms}, 18 \mathrm{~ms}$, and $15 \mathrm{~ms}$, respectively. Thus, we can opt for giving up a certain level of accuracy as a trade-off for higher efficiency. Note that PDA-LSH achieves a better trade-off between efficiency and accuracy than QALSH and SRS do. For example, on Msong dataset, when $c=1.5$, the I/O cost and running time of PDA-LSH are comparable to that of QALSH with $c=2.0$, yet the overall ratio of PDA-LSH is much lower. Compared with SRS, by setting $c=3.0$, the overall ratio of PDA-LSH is comparable to that of SRS with $c=2.0$, yet PDA-LSH has much less running time.

\section{Conclusion}

In this paper, we address the problem of indexing high-dimensional streaming data for efficient $c$-ANN search, in which case the update efficiency, search efficiency, and quality guarantees are crucial. Previous disk-based LSH schemes mainly focused on reducing the search cost while preserving an acceptable accuracy, but often at the expense of high index maintenance overhead or 
abandoning the quality guarantees. In view of this, We propose a novel solution, PDA-LSH, to support efficient searches and updates. The key insight is (1) to employ write-friendly LSM-trees for indexing and (2) to make full use of the precise projected distances. Using a novel scheme of estimation, PDA-LSH requires less number of LSH functions than the start-of-art solutions do. As a result, both index size and index access cost are reduced. With a systematic way of setting its parameters, PDA-LSH guarantees the success probability. Experiments have demonstrated the effectiveness and efficiency of PDA-LSH.

\section{References}

1. Roger Weber, Hans-Jörg Schek, and Stephen Blott. A quantitative analysis and performance study for similarity-search methods in high-dimensional spaces. In $V L D B$, pages 194-205, 1998.

2. Piotr Indyk and Rajeev Motwani. Approximate nearest neighbors: Towards removing the curse of dimensionality. In STOC, pages 604-613, 1998.

3. Qi Li, Zhenan Sun, Ran He, and Tieniu Tan. Deep supervised discrete hashing. In NIPS, pages 2482-2491, 2017.

4. Jinyang Gao, Hosagrahar Visvesvaraya Jagadish, Wei Lu, and Beng Chin Ooi. DSH: Data sensitive hashing for high-dimensional k-NN search. In SIGMOD, pages $1127-$ 1138, 2014.

5. Kang Zhao, Hongtao Lu, and Jincheng Mei. Locality preserving hashing. In $A A A I$, pages $2874-2881,2014$.

6. Jinyang Gao, H. V. Jagadish, Beng Chin Ooi, and Sheng Wang. Selective hashing: Closing the gap between radius search and k-nn search. In SIGKDD, pages 349-358, 2015.

7. Qin Lv, William Josephson, Zhe Wang, Moses Charikar, and Kai Li. Multi-probe LSH: efficient indexing for high-dimensional similarity search. In $V L D B$, pages 950-961, 2007.

8. Junhao Gan, Jianlin Feng, Qiong Fang, and Wilfred Ng. Locality-sensitive hashing scheme based on dynamic collision counting. In SIGMOD, pages 541-552, 2012.

9. Qiang Huang, Jianlin Feng, Qiong Fang, Wilfred Ng, and Wei Wang. Query-aware locality-sensitive hashing scheme for lp norm. VLDB J., 26(5):683-708, 2017.

10. Yifang Sun, Wei Wang, Jianbin Qin, Ying Zhang, and Xuemin Lin. SRS: solving capproximate nearest neighbor queries in high dimensional euclidean space with a tiny index. PVLDB, 8(1):1-12, 2014.

11. João Gama, Raquel Sebastião, and Pedro Pereira Rodrigues. On evaluating stream learning algorithms. Mach. Learn., 90(3):317-346, 2013.

12. Tingting Zhai, Yang Gao, Hao Wang, and Longbing Cao. Classification of highdimensional evolving data streams via a resource-efficient online ensemble. Data Min. Knowl. Disc., 31:1242-1265, 2017.

13. Alexandr Andoni, Piotr Indyk, Thijs Laarhoven, Ilya P. Razenshteyn, and Ludwig Schmidt. Practical and optimal LSH for angular distance. In NIPS, pages 1225-1233, 2015.

14. Kave Eshghi and Shyamsundar Rajaram. Locality sensitive hash functions based on concomitant rank order statistics. In SIGKDD, pages 221-229, 2008.

15. Mayur Datar, Nicole Immorlica, Piotr Indyk, and Vahab S. Mirrokni. Locality-sensitive hashing scheme based on p-stable distributions. In SoCG, pages 253-262, 2004.

16. Yufei Tao, Ke Yi, Cheng Sheng, and Panos Kalnis. Efficient and accurate nearest neighbor and closest pair search in high-dimensional space. ACM Trans. Database Syst., 35(3):20:1-20:46, 2010.

17. Yuxin Zheng, Qi Guo, Anthony K. H. Tung, and Sai Wu. LazyLSH: Approximate nearest neighbor search for multiple distance functions with a single index. In SIGMOD, pages 2023-2037, 2016.

18. Rina Panigrahy. Entropy based nearest neighbor search in high dimensions. In $S O D A$, pages 1186-1195, 2006. 
19. Yingfan Liu, Jiangtao Cui, Zi Huang, Hui Li, and Heng Tao Shen. SK-LSH: an efficient index structure for approximate nearest neighbor search. PVLDB, 7(9):745-756, 2014.

20. Chaoqun Chu, Dahan Gong, Kai Chen, Yuchen Guo, Jungong Han, and Guiguang Ding. Optimized projection for hashing. Pattern Recognition Letters, 117:169-178, 2019.

21. Xingbo Liu, Xiushan Nie, Yingxin Wang, and Yilong Yin. Jointly multiple hash learning. In $A A A I$, pages 9981-9982, 2019.

22. Niv Dayan, Manos Athanassoulis, and Stratos Idreos. Monkey: Optimal navigable keyvalue store. In SIGMOD, pages 79-94, 2017.

23. Wanqi Liu, Hanchen Wang, Ying Zhang, Wei Wang, and Lu Qin. I-LSH: I/O efficient c-approximate nearest neighbor search in high-dimensional space. In ICDE, pages 16701673, 2019.

24. Hervé Jégou, Matthijs Douze, and Cordelia Schmid. Product quantization for nearest neighbor search. IEEE Trans. Pattern Anal. Mach. Intell., 33(1):117-128, 2011.

\section{Appendix A: Sketch of Proof of Lemma 1}

In this section, we provide a brief proof of Lemma 1 in Section 4.3. Recall that, for arbitrary $\boldsymbol{o}_{1}, \boldsymbol{o}_{2}, \boldsymbol{q} \in \mathbb{R}^{d}$, Lemma 1 asserts the following:

$$
\begin{aligned}
\operatorname{Pr}\left[\mathbf{E}_{1}\left(s_{1}, R\right)\right] & >\operatorname{Pr}\left[\mathbf{E}_{1}\left(s_{2}, R\right)\right], \\
\operatorname{Pr}\left[\mathbf{E}_{2}\left(s_{1}, R\right) \mid \mathbf{E}_{1}\left(s_{1}, R\right)\right] & >\operatorname{Pr}\left[\mathbf{E}_{2}\left(s_{2}, R\right) \mid \mathbf{E}_{1}\left(s_{2}, R\right)\right], \\
\operatorname{Pr}\left[\mathbf{E}_{0}\left(s_{1}, R\right)\right] & >\operatorname{Pr}\left[\mathbf{E}_{0}\left(s_{2}, R\right)\right],
\end{aligned}
$$

where $s_{1}=\operatorname{dist}\left(\boldsymbol{o}_{1}, \boldsymbol{q}\right), s_{2}=\operatorname{dist}\left(\boldsymbol{o}_{2}, \boldsymbol{q}\right)$, and $s_{1}<s_{2}$.

To prove Lemma 1 , we need to consider the following functions:

$$
\begin{aligned}
F(k, m, x) & \stackrel{\text { def }}{=} \sum_{i=0}^{k}\left(\begin{array}{c}
m \\
i
\end{array}\right) x^{i}(1-x)^{m-i}, \\
g_{i}(n, m, x) & \stackrel{\text { def }}{=} \frac{\left(\begin{array}{c}
m \\
i
\end{array}\right) x^{i}(1-x)^{m-i}}{1-F(n-1, m, x)}, \\
G\left(n, n^{\prime}, m, x\right) & \stackrel{\text { def }}{=} \sum_{i=n}^{n^{\prime}} g_{i}(n, m, x) \\
& =\frac{F\left(n^{\prime}, m, x\right)-F(n-1, m, x)}{1-F(n-1, m, x)} .
\end{aligned}
$$

The following several technical lemmata are useful.

Lemma $3 F(k, m, x)$ is monotonically decreasing with respect to $x$ if $0 \leq x \leq 1$ and $k<m$.

Lemma $4 G\left(n, n^{\prime}, m, x\right)$ is monotonically decreasing with respect to $x$ if $0 \leq x \leq 1$ and $n \leq n^{\prime}<m$.

Lemmata 3 and 4 state some monotonicity properties of the binomial distribution, of which the proofs are pure mathematical and are essentially unsophisticated. Therefore, we omit their proofs to avoid further distraction from our main focus.

We are now ready to present the proof of Lemma 1.

Proof (Sketch of Proof of Lemma 1) Recall that we have established

$$
\begin{aligned}
\operatorname{Pr}\left[\mathbf{E}_{1}(s, R)\right] & =\sum_{i=L}^{|\mathcal{H}|}\left(\begin{array}{c}
|\mathcal{H}| \\
i
\end{array}\right) p(s, R)^{i}(1-p(s, R))^{|\mathcal{H}|-i}, \\
& =1-F(L-1,|\mathcal{H}|, p(s, R)),
\end{aligned}
$$


where $p(s, R)=2 \Phi\left(\frac{w R}{2 s}\right)-1$ (i.e., Equation 3). Since $p(s, R)$ is monotonically decreasing with respect to $s$, it is clear that, according to Lemma $3, \operatorname{Pr}\left[\mathbf{E}_{1}(s, R)\right]$ is monotonically decreasing with respect to $s$, which proves Inequality 4 .

To prove Inequality 5 , note that

$$
\begin{aligned}
\operatorname{Pr} & {\left[\mathbf{E}_{2}(s, R) \mid \mathbf{E}_{1}(s, R)\right] } \\
& =\sum_{i=L}^{m} \frac{\operatorname{Pr}[\operatorname{Col}(\boldsymbol{o}, R)=i]}{\operatorname{Pr}\left[\mathbf{E}_{1}(s, R)\right]} \operatorname{Pr}\left[\mathbf{E}_{2}(s, R) \mid \operatorname{Col}(\boldsymbol{o}, R)=i\right] \\
& =\sum_{i=L}^{m} g_{i}(L,|\mathcal{H}|, p(s, R)) \operatorname{Pr}\left[\mathbf{E}_{2}(s, R) \mid \operatorname{Col}(\boldsymbol{o}, R)=i\right] .
\end{aligned}
$$

Consider $g_{i}(L,|\mathcal{H}|, p(s, R))$ as a distribution over the number of collisions $i=L, L+1, \cdots, m$. The monotonicity of

$$
G\left(L, L^{\prime},|\mathcal{H}|, p(s, R)\right)=\sum_{i=L}^{L^{\prime}} g_{i}(L,|\mathcal{H}|, p(s, R)),
$$

as stated in Lemma 4 , implies that $g_{i}(L,|\mathcal{H}|, p(s, R))$ is more skewed towards small $i$ 's as $s$ increases. In addition, it can be proved that the probability $\operatorname{Pr}\left[\mathbf{E}_{2}(s, R) \mid \operatorname{Col}(\boldsymbol{o}, R)=i\right]$, viewed as a function of $i$ and $s$, is monotonically decreasing with respect to $s$ and monotonically increasing with respect to $i$. Combining the above results, we obtain

$$
\begin{aligned}
\operatorname{Pr} & {\left[\mathbf{E}_{2}\left(s_{1}, R\right) \mid \mathbf{E}_{1}\left(s_{1}, R\right)\right] } \\
& =\sum_{i=L}^{m} g_{i}\left(L,|\mathcal{H}|, p\left(s_{1}, R\right)\right) \operatorname{Pr}\left[\mathbf{E}_{2}\left(s_{1}, R\right) \mid \operatorname{Col}(\boldsymbol{o}, R)=i\right] \\
& >\sum_{i=L}^{m} g_{i}\left(L,|\mathcal{H}|, p\left(s_{2}, R\right)\right) \operatorname{Pr}\left[\mathbf{E}_{2}\left(s_{1}, R\right) \mid \operatorname{Col}(\boldsymbol{o}, R)=i\right] \\
& >\sum_{i=L}^{m} g_{i}\left(L,|\mathcal{H}|, p\left(s_{2}, R\right)\right) \operatorname{Pr}\left[\mathbf{E}_{2}\left(s_{2}, R\right) \mid \operatorname{Col}(\boldsymbol{o}, R)=i\right] \\
& =\operatorname{Pr}\left[\mathbf{E}_{2}\left(s_{2}, R\right) \mid \mathbf{E}_{1}\left(s_{2}, R\right)\right] .
\end{aligned}
$$

Finally, Inequality 6 is a direct corollary of Inequalities 4 and 5 since $\mathbf{E}_{0}(s, R)=$ $\mathbf{E}_{1}(s, R) \cap \mathbf{E}_{2}(s, R)$ by definition. 